\title{
Editorial for special section on ICNC-FSKD 2017
}

\author{
Lipo Wang ${ }^{1}$
}

Published online: 3 March 2020

(C) Springer-Verlag GmbH Germany, part of Springer Nature 2020
This special section is dedicated to the 2017 13th International Conference on Natural Computation, Fuzzy Systems and Knowledge Discovery (ICNC-FSKD 2017), 29-31 July 2017, Guilin, China. ICNC-FSKD has been a premier international forum for scientists and researchers to present the state of the art of soft computing techniques and applications. This special section includes the following four papers.

Huaping Guo et al. proposed an ensemble learning via constraint projection and under-sampling technique for class-imbalance problems. The authors construct each base classifier in two steps. First, they construct a set of pairwise constraints by under-sampling examples from the minority/majority class set and learning a projection matrix from the pairwise constraint set. Second, they under-sample the original training set to obtaining a new training set on which a base classifier is constructed in the new feature space defined by the projection matrix. The projection matrix is used to enhance separability between diverse class examples and to improve the performance of the base classifier, while under-sampling creates various sets of pairwise constraints to train projection matrices, thereby inducing diversity to the base classifiers. The authors show experimentally that the proposed method leads to significantly better performance on a variety of measures, compared to other state-of-the-art methods for 29 datasets with different degrees of data imbalance.

Yaoli Wang et al. investigated effects of direct input-tooutput connections on multilayer perceptron (MLP) neural networks for time series prediction. Eight different time series datasets were used to verify the validity of backpropagation neural networks with direct input-to-output connections (BPNN-DIOC). The authors extend the BPNN to four variants based on the presence or absence of the output layer bias and the direct input-to-output connections. Statistic methods are used to analyze and rank the prediction

Lipo Wang

ELPWang@ntu.edu.sg

1 School of Electrical and Electronic Engineering, Nanyang Technological University, Block S1, Nanyang Avenue, Singapore 639798, Singapore accuracy results. Extensive experiments demonstrate that the BPNN-DIOC has better prediction accuracy compared the conventional BPNN, while the output layer bias has no significant effect. The authors thus conclude that the direct input-to-output connections can significantly improve neural network prediction abilities for time series.

Shike Zhang et al. presented an interesting application of soft computing for earth stress analysis in hydropower engineering. The authors employ an artificial neural network (ANN) to map the relationship between the earth stress parameters and hydraulic fracturing behaviour. The advantages of this approach include: (1) the approach can conveniently integrate the ANN with optimization algorithms and (2) the approach can effectively reduce the workload of numerical computation by using directly the field measured information to build learning samples. The ANN model is able to improve accuracy of earth stress determination from field test data. Genetic algorithms (GA) are used to implement multi-objective optimization on the earth stress parameters. Field monitoring information in a real hydropower project is used to verify the proposed soft computing method.

Jiancheng An et al. studied grayscale images colorization with convolutional neural networks. Previous methods for colorization of grayscale images rely on manual labor and often produce results different from true colorization. Inspired by the work of Richart and Visca, the authors propose an automatic approach based on deep neural networks to color grayscale images. The authors explore several models, techniques, and loss functions, in search for the best approaches to producing a plausible colorization. Through extensive experiments, they concluded that the VGG-16 CNN model based on classification with the loss of cross-entropy shows optimal performance.

Our gratitude goes to the authors for contributing their work to this special section and the reviewers for their time and efforts. We would also like to thank Professor Vincezo Loia for approving this special section and offering help and support along the way.

Publisher's Note Springer Nature remains neutral with regard to jurisdictional claims in published maps and institutional affiliations. 